\title{
Curves which do not Become Semi-Stable After any Solvable Extension
}

\begin{abstract}
Ambrus PÁl (*)
ABstract - We show that there is a field $F$ complete with respect to a discrete valuation whose residue field is perfect and there is a finite Galois extension $K \mid F$ such that there is no solvable Galois extension $L \mid F$ such that the extension $K L \mid K$ is unramified, where $K L$ is the composite of $K$ and $L$. As an application we deduce that that there is a field $F$ as above and there is a smooth, projective, geometrically irreducible curve over $F$ which does not acquire semi-stable reduction over any solvable extension of $F$.
\end{abstract}

Mathematics Subject Classification (2010). 11G25, $11 \mathrm{G} 10$.

KEYwords. Local fields, abelian varieties, semistable reduction.

\section{Introduction}

We say that a finite Galois field extension $K \mid F$ is solvable if its Galois group is solvable. In this paper we will show the following:

THEOREM 1.1. There is a field $F$ of characteristic zero complete with respect to a discrete valuation whose residue field is perfect and there is a finite Galois extension $K \mid F$ such that there is no solvable Galois extension $L \mid F$ such that the extension $K L \mid K$ is unramified, where $K L$ is the composite of $K$ and $L$.

This result gives a counterexample to Lemma 6.4 of [9] on page 632. Its proof is also quite intricate, for example it uses some non-trivial facts about

(*) Indirizzo dell'A.: Department of Mathematics, 180 Queen's Gate, Imperial College, London SW7 2AZ, United Kingdom.

E-mail: a.pal@imperial.ac.uk 
Drinfeld modular curves. However it is mainly interesting because it has the following

COROLlaRY 1.2. There is a field $F$ of characteristic zero complete with respect to a discrete valuation whose residue field is perfect and there is a smooth, projective, geometrically irreducible curve over $F$ which does not acquire semi-stable reduction over any solvable extension of $F$.

This result is significant because it opens up the possibility to go beyond the results of my paper [9] in its quest to construct curves of a given genus without solvable points. In a joint paper [6] Gyula Károlyi and I determined the set of natural numbers for which the original method of [9] can work which uses curves with semi-stable reduction. This set does not contain the number 23, for example, but it looks unlikely that every genus 23 curve has a solvable point. However it might be possible to construct counter-examples with this genus by using a curve which will not acquire semi-stable reduction over any solvable extension.

The contents of this paper are the following. In the next section we prove Theorem 1.1. In the third section we first give an overview of Gerritzen's uniformisation theory of abelian varieties by non-archimedean tori, then we derive Corollary 1.2.

\section{Extensions whose ramification does not disappear after any solva- ble extension}

Notation 2.1. For every field $K$ let $\bar{K}$ denote its separable closure. We say that a field $F$ is local if it is complete with respect to a discrete valuation. For the sake of simplicity we will assume that every local field in this paper has perfect residue field. Let $F$ be a local field of characteristic zero whose residue field $\boldsymbol{k}_{F}$ has characteristic $p>0$. For every such $F$ let $\Gamma_{F}$ and $I_{F}$ denote the absolute Galois group of $F$ and the inertia subgroup of $\Gamma_{F}$, respectively. For every $\mathbb{F}_{p}\left[S L_{2}\left(\mathbb{F}_{p}\right)\right]$-module $M$ we say that a group $G$ is $M$-type if it is the extension of $S L_{2}\left(\mathbb{F}_{p}\right)$ by $M$ and the action of $S L_{2}\left(\mathrm{~F}_{p}\right)$ on $M$ via conjugation in $G$ is the given one. Given two Galois extensions $K \mid F$ and $L \mid F$ let $K L \mid F$ denote their composite.

Lemma 2.2. Assume that $p \geq 5$ and let $L \mid F$ be a finite Galois extension whose Galois group is $M$-type for an $\mathbb{F}_{p}\left[S L_{2}\left(\mathbb{F}_{p}\right)\right]$-module $M$. Also suppose that the image of $I_{F}$ in $\operatorname{Gal}(L \mid F)$ is an $\mathbb{F}_{p}\left[S L_{2}\left(\mathbb{F}_{p}\right)\right]$-submodule $N \leq M$ 
which is isomorphic to $\mathbb{F}_{p}^{2}$ with its usual $S L_{2}\left(\mathbb{F}_{p}\right)$-action. Then for every finite solvable Galois extension $P \mid F$ the image of $I_{P}$ in $\operatorname{Gal}(L P \mid P) \leq$ $\operatorname{Gal}(L \mid F)$ is also $N$.

Proof. Note that $\operatorname{Gal}(L P \mid P)$ is a normal subgroup of $\operatorname{Gal}(L \mid F)$ whose quotient subgroup is isomorphic to $\operatorname{Gal}(P \mid F)$, and hence the image of $\operatorname{Gal}(L P \mid P)$ in the quotient $S L_{2}\left(\mathbb{F}_{p}\right)$ of $\operatorname{Gal}(L \mid F)$ by $M$ is also a normal subgroup with a solvable quotient. Since the only such subgroup of $S L_{2}\left(\mathbb{F}_{p}\right)$ is itself we get that $\operatorname{Gal}(L P \mid P)$ surjects onto the quotient $S L_{2}\left(\mathrm{~F}_{p}\right)$ of $\operatorname{Gal}(L \mid F)$. This means that we may reduce to the case when $\operatorname{Gal}(P \mid F)$ is a group whose order is a prime $l$ by induction on the order of $\operatorname{Gal}(P \mid F)$. The quotient group $I_{F} / I_{P}$ is isomorphic to $\operatorname{Gal}\left(P^{u n} \mid F^{u n}\right)$ where $F^{u n}$ and $P^{u n}$ are the maximal unramified extensions of $F$ and $P$, respectively. Since $\operatorname{Gal}\left(P^{u n} \mid F^{u n}\right)$ is a subgroup of $\operatorname{Gal}(P \mid F)$ we get that the index of $I_{P}$ in $I_{F}$ divides $l$. Therefore the image $R$ of $I_{P}$ in $\operatorname{Gal}(L P \mid P)$ is a normal subgroup of $N$ of index dividing $l$. Moreover $R$ is a normal subgroup in $\operatorname{Gal}(L P \mid P)$ so it is invariant under the action of $S L_{2}\left(\mathrm{~F}_{p}\right)$. Because the action of $S L_{2}\left(\mathbb{F}_{p}\right)$ on $N$ is irreducible we get that $R$ is equal to $N$.

Notation 2.3. For every local field $F$ as above let $\mathcal{O}_{F}$ and $\boldsymbol{k}_{F}$ denote its valuation ring and its residue field, respectively. Let $\operatorname{ord}_{F}$ denote the valuation of $F$ normalised such that $\operatorname{ord}_{F}(\pi)=1$ for every uniformizer $\pi \in \mathcal{O}_{F}$. Let $e=\operatorname{ord}_{F}(p)$ denote the absolute ramification index of $F$. For the sake of simple notation let $U_{F}=\mathcal{O}_{F}^{*}$ and for every $i \in \mathbb{N}$ let

$$
U_{F}^{(i)}=\left\{u \in U_{F} \mid \operatorname{ord}_{F}(1-u) \geq i\right\} .
$$

In the next three lemmas we will assume that $F$ contains the $p$-th roots of unity. Let $m=e /(p-1)$.

LEMMA 2.4. Every extension $K \mid F$ which we get by adjoining a $p$-th root of any $\alpha \in U_{F}^{(m p)}$ is unramified.

Proof. We may assume without the loss of generality that $\alpha$ is not a $p$ th root in $F$. Let $\beta \in K$ be a $p$-th root of $\alpha$ and fix a uniformizer $\pi \in F$. Then $\gamma=\pi^{-m}(\beta-1)$ generates the degree $p$ extension $K \mid F$. The minimal polynomial of this element is:

$f(x)=\pi^{-m p}\left(\left(\pi^{m} x+1\right)^{p}-\alpha\right)=\pi^{-m p}(1-\alpha)+\sum_{k=1}^{p-1}\left(\begin{array}{l}p \\ k\end{array}\right) \pi^{m(k-p)} x^{k}+x^{p} \in F[x]$. 
This is a monic polynomial whose constant term $\pi^{-m p}(1-\alpha)$ is in $\mathcal{O}_{F}$ by assumption. For every integer $k$ with $1 \leq k \leq p-1$ we have:

$$
\operatorname{ord}_{F}\left(\left(\begin{array}{l}
p \\
k
\end{array}\right) \pi^{m(k-p)}\right)=m(p-1)+m(k-p)=m(k-1) \geq 0,
$$

so $f(x) \in \mathcal{O}_{F}[x]$. Let $L \mid F$ be the unique unramified Galois extension such that $\boldsymbol{k}_{L}$ is the splitting field of $f(x)$ modulo $\pi \mathcal{O}_{F}$. Since $f^{\prime}(x) \equiv p \pi^{m(1-p)} \not \equiv 0 \bmod \pi \mathcal{O}_{F}$ the polynomial $f(x)$ splits in $L$ by Hensel's lemma. Therefore $K=F(\gamma)$ is contained in $L$, and hence it is an unramified extension of $F$ as we claimed.

Lemma 2.5. We have $\left(F^{*}\right)^{p} \cap U_{F}^{(m p-1)} \subseteq U_{F}^{(m p)}$.

Proof. Fix a uniformizer $\pi \in \mathcal{O}_{F}$ and let $x \in F^{*}$ such that $x^{p} \in U_{F}^{(m p-1)}$. Clearly we have $x \in U_{F}^{(1)}$ and hence there is a positive integer $n$ and an $x_{0} \in \mathcal{O}_{F}^{*}$ such that $x=1+\pi^{n} x_{0}$. By the binomial theorem:

$$
x^{p}=\left(1+\pi^{n} x_{0}\right)^{p}=1+p \pi^{n} x_{0}+\cdots+\left(\begin{array}{l}
p \\
k
\end{array}\right) \pi^{n k} x_{0}^{k}+\cdots+\pi^{n p} x_{0}^{p} .
$$

Assume first that $n<m$. Then for every integer $k$ with $1 \leq k \leq p-1$ we have:

$$
\operatorname{ord}_{F}\left(\pi^{n p} x_{0}^{p}\right)=p n<(p-1) m+n k=\operatorname{ord}_{F}\left(\left(\begin{array}{l}
p \\
k
\end{array}\right) \pi^{n k} x_{0}^{k}\right),
$$

and hence $x^{p} \notin U_{F}^{(m p-1)}$. This is a contradiction, so $n \geq m$. In this case

$$
p m \leq \operatorname{ord}_{F}\left(\pi^{n p} x_{0}^{p}\right) \text { and } p m \leq \operatorname{ord}_{F}\left(\left(\begin{array}{l}
p \\
k
\end{array}\right) \pi^{n k} x_{0}^{k}\right),
$$

and hence $x^{p} \in U_{F}^{(m p)}$ as we claimed.

For every group $G$ and for every $\mathbb{F}_{p}[G]$-module $N$ let $N^{\vee}$ denote its dual $\mathbb{F}_{p}[G]$-module $\operatorname{Hom}_{\mathbb{F}_{p}}\left(N, \mathbb{F}_{p}\right)$. (Recall that for a left $\mathbb{F}_{p}[G]$-module $N$ we define the left $G$-multiplication on $N^{\vee}$ by the formula $g \lambda(x)=\lambda\left(g^{-1} x\right)$ for every $g \in G, \lambda \in \operatorname{Hom}_{\mathbb{F}_{p}}\left(N, \mathbb{F}_{p}\right)$ and $x \in N$.)

LEMma 2.6. Let $K \mid F$ be a finite unramified Galois extension with Galois group $S L_{2}\left(\mathbb{F}_{p}\right)$. Let $N \leq \boldsymbol{k}_{K}$ be an irreducible finite $\mathbb{F}_{p}\left[\operatorname{Gal}\left(\boldsymbol{k}_{K} \mid \boldsymbol{k}_{F}\right)\right]=$ $\mathbb{F}_{p}[\operatorname{Gal}(K \mid F)]$-module. Then there is a finite Galois extension $L \mid F$ such that

(i) the group $\operatorname{Gal}(L \mid F)$ is $M$-type for a finite $\mathbb{F}_{p}\left[S L_{2}\left(\mathbb{F}_{p}\right)\right]$-module $M$ which contains $N^{\vee}$,

(ii) the field $L$ contains $K$ and $M \cong \operatorname{Gal}(L \mid K)$,

(iii) the image of $I_{F}$ in $M \leq \operatorname{Gal}(L \mid F)$ is $N^{\vee}$. 
Proof. Fix a uniformizer $\pi \in \mathcal{O}_{F}$. Then the homomorphism $\mathcal{O}_{K} \rightarrow$ $U_{K}^{(m p-1)}$ given by the rule $x \mapsto 1+x \pi^{m p-1}$ maps $\pi \mathcal{O}_{F}$ onto $U_{F}^{(m p)}$ and hence induces an isomorphism:

$$
\boldsymbol{k}_{K} \cong \mathcal{O}_{K} / \pi \mathcal{O}_{K} \cong U_{K}^{(m p-1)} / U_{K}^{(m p)} .
$$

This isomorphism is $\operatorname{Gal}(K \mid F)$-equivariant where we consider $\boldsymbol{k}_{K}$ as a $\operatorname{Gal}(K \mid F)$-module via the natural action of $\operatorname{Gal}\left(\boldsymbol{k}_{K} \mid \boldsymbol{k}_{F}\right)$ and we equip $U_{K}^{(m p-1)} / U_{K}^{(m p)}$ with the quotient $\operatorname{Gal}(K \mid F)$-module structure. By applying Lemma 2.5 to the local field $K$ we get that there is a natural quotient map:

$$
q: U_{K}^{(m p-1)} / U_{K}^{(m p-1)} \cap\left(K^{*}\right)^{p} \rightarrow U_{K}^{(m p-1)} / U_{K}^{(m p)} .
$$

Choose a finite set $\bar{N} \subset U_{K}^{(m p-1)} / U_{K}^{(m p-1)} \cap\left(K^{*}\right)^{p}$ such that the image of $\bar{N}$ under $q$ is $N$. Let $M_{0} \subseteq U_{K}^{(m p-1)} / U_{K}^{(m p-1)} \cap\left(K^{*}\right)^{p}$ be the $\mathbb{F}_{p}[\operatorname{Gal}(K \mid F)]$ module generated by $\bar{N}$. Since $M_{0}$ is the $\mathbb{F}_{p}$-span of all the $\operatorname{Gal}(K \mid F)$ conjugates of $\bar{N}$, and since that set is finite, we get that $M_{0}$ is a finite $\mathbb{F}_{p}[\operatorname{Gal}(K \mid F)]$-module. Let $\bar{M} \subseteq U_{K}^{(m p-1)}$ be a set of representatives of $M_{0}$ and let $L \mid K$ be the finite Galois extension which we get by adjoining the $p$-th roots of $\bar{M}$. This extension is independent of the choice of $\bar{M}$ and since $M_{0}$ is invariant under the action of $\operatorname{Gal}(K \mid F)$ the extension $L \mid F$ is also Galois. By Kummer theory $\operatorname{Gal}(L \mid K)$ is isomorphic to $M=M_{0}^{\vee}$ as a $\operatorname{Gal}(K \mid F)$-module. The image of $M_{0}$ with respect to $q$ is $N$ and hence $M_{0}$ has a quotient $\mathbb{F}_{p}[\operatorname{Gal}(K \mid F)]$-module isomorphic to $N$. By duality $M$ has a $\mathbb{F}_{p}[\operatorname{Gal}(K \mid F)]$-submodule isomorphic to $N^{\vee}$. So properties (i) and (ii) hold for $L \mid F$.

Let $M_{0}^{\prime}$ be the kernel of the restriction of $q$ onto $M_{0}$ and let $\bar{M}^{\prime} \subseteq U_{K}^{(m p)}$ be a set of representatives of $M_{0}^{\prime}$. Let $L^{\prime} \mid K$ be the the finite Galois extension which we get by adjoining the $p$-th roots of $\bar{M}^{\prime}$. By Lemma 2.4 the extension $L^{\prime} \mid K$ is unramified. Since the extension $K \mid F$ is also unramified we get that $L^{\prime} \mid F$ is an unramified extension, too. Because $N^{\vee} \leq \operatorname{Gal}(L \mid F)$ is equal to the Galois group $\operatorname{Gal}\left(L \mid L^{\prime}\right)$, the image of $I_{F}$ in $\operatorname{Gal}(L \mid F)$ is contained in $N^{\vee}$. By Lemma 2.5 there is not any unramified extension of $K$ where any element of $U_{K}^{(m p-1)}-U_{K}^{(m p)}$ could be a $p$-th root. Therefore the extension $L \mid K$ is ramified and so the image of $I_{F}$ in $N^{\vee}$ is non-trivial. Because $N^{\vee}$ is the dual of an irreducible $\mathbb{F}_{p}[\operatorname{Gal}(K \mid F)]$-module, it is also irreducible and hence the image of $I_{F}$ must be $N^{\vee}$ itself.

Proposition 2.7. Let $\boldsymbol{f}$ be an infinite perfect field of characteristic $p$ whose Brauer group has trivial 2-torsion and let $\boldsymbol{k} \mid \boldsymbol{f}$ be a finite Galois 
extension. Let $N$ be an $\mathbb{F}_{p}[\operatorname{Gal}(\boldsymbol{k} \mid \boldsymbol{f})]$-module which has dimension two as a vector space over $\mathbb{F}_{p}$. Then there is a $\mathbb{F}_{p}[\mathrm{Gal}(\boldsymbol{k} \mid \boldsymbol{f})]$-submodule of $\boldsymbol{k}$ which is isomorphic to $N$.

Proof. Note that it will be enough to show that there is an $\mathbb{F}_{p}[\operatorname{Gal}(\overline{\boldsymbol{f}} \mid \boldsymbol{f})]$-submodule $M$ of $\overline{\boldsymbol{f}}$ which is isomorphic to $N$ as an $\mathbb{F}_{p}[\operatorname{Gal}(\overline{\boldsymbol{f}} \mid \boldsymbol{f})]$ module. In fact in this case the action of $\operatorname{Gal}(\overline{\boldsymbol{f}} \mid \boldsymbol{f})$ on the extension of $\boldsymbol{f}$ generated by $M$ factors through Gal $(\boldsymbol{k} \mid \boldsymbol{f})$. Therefore $M$ will be a submodule of $\boldsymbol{k}$ by the fundamental theorem of Galois theory. Let $A$ denote the polynomial ring $\mathbb{F}_{p}[t]$. We may consider $\boldsymbol{f}$ as an extension of the residue field $\mathbb{F}_{p}=A /(t+1)$ of the prime ideal $(t+1) \triangleleft A$ and hence we may talk about Drinfeld $A$-modules of rank 2 of characteristic $(t+1)$ with coefficients in $\boldsymbol{f}$. For such a Drinfeld $A$-module $\phi$ its $t$-torsion group scheme $\phi[t]$ is étale and hence $\phi[t](\overline{\boldsymbol{f}})$ is a $\mathbb{F}_{p}[\mathrm{Gal}(\overline{\boldsymbol{f}} \mid \boldsymbol{f})]$-submodule of $\overline{\boldsymbol{f}}$ which has dimension two as a vector space over $\mathbb{F}_{p}$.

Let $Y(t)$ be the Drinfeld modular curve over $\mathbb{F}_{p}=A /(t+1)$ which parameterizes Drinfeld $A$-modules of rank 2 of characteristic $(t+1)$ with a full $t$-level structure. By fixing an $\mathbb{F}_{p}$-basis of $N$ we get a continuous Galois representation $\rho: \operatorname{Gal}(\overline{\boldsymbol{f}} \mid \boldsymbol{f}) \rightarrow G L_{2}\left(\mathbb{F}_{p}\right)$. Let $Y_{\rho}$ be the twist of $Y(t)$ with respect to $\rho$ via the natural action of $G L_{2}\left(\mathrm{~F}_{p}\right)$ on $Y(t)$. The $\boldsymbol{f}$-valued rational points of $Y_{\rho}$ correspond to Drinfeld $A$-modules of rank 2 of characteristic $(t+1)$ with coefficients in $\boldsymbol{f}$ such that the Gal $(\overline{\boldsymbol{f}} \mid \boldsymbol{f})$-module $\phi[t](\overline{\boldsymbol{f}})$ is isomorphic to $N$. Since the smooth compatification of the affine curve $Y(t)$ is geometrically irreducible of genus zero, the same holds for $Y_{\rho}$. As the 2torsion of the Brauer group of $\boldsymbol{f}$ is zero we get that $Y_{\rho}$ has a Zariski-dense set of $\boldsymbol{f}$-valued points.

Proof of Theorem 1.1. Let $p \geq 5$ and let $\boldsymbol{f}$ be the perfection of a function field of transcendence degree one over an algebraically closed field of characteristic $p$. Then there is a finite Galois extension $\boldsymbol{k} \mid \boldsymbol{f}$ with Galois group $S L_{2}\left(\mathrm{~F}_{p}\right)$ by Harbater's theorem (see Corollary 1.5 of [5] on page 284). Let $F$ be the field which we get by adjoining the $p$-th roots of unity to the fraction field of the ring of Witt vectors of $\boldsymbol{f}$ and let $K$ be the unique unramified extension of $F$ with residue field $\boldsymbol{k}$. By Tsen's theorem the Brauer group of $\boldsymbol{f}$ is trivial and hence there is a $\operatorname{Gal}(\boldsymbol{k} \mid \boldsymbol{f})$-submodule $N \leq \boldsymbol{k}_{K}$ which is isomorphic to $\mathbb{F}_{p}^{2}$ with its usual $S L_{2}\left(\mathbb{F}_{p}\right)$-action by Proposition 2.7. Because $\mathbb{F}_{p}^{2}$ is self-dual as an $\mathbb{F}_{p}\left[S L_{2}\left(\mathbb{F}_{p}\right)\right]$-module we may apply Lemma 2.6 to the extension $K \mid F$ and the module $N$ to get a finite Galois extension $L \mid F$ which satisfies the conditions in Lemma 2.2. The claim now follows from this lemma. 
Curves which do not Become Semi-Stable After any Solvable Extension 271

\section{Abelian varieties which do not become semi-stable after any solvable extension}

Definition 3.1. For every algebraic group $T$ over a field $F$ which is a split torus, let $C(T)$ denote its group of cocharacters. Then $C(T)$ is a free and finitely generated abelian group whose rank is equal to the dimension of $T$ over $F$. The group $T(F)$ of $F$-valued points of $T$ is canonically isomorphic to $F^{*} \otimes C(T)$. Now let $F$ be a field complete with respect to a discrete valuation $v: F^{*} \rightarrow Z$. A subgroup $\Lambda$ of $T(F)=F^{*} \otimes C(T)$ is called a discrete lattice if the restriction of the homomorphism $v \otimes 1: F^{*} \otimes C(T) \rightarrow Z \otimes C(T)$ to $\Lambda$ is injective and it has finite cokernel. In this case the quotient $T / \Lambda$ exists in the category of rigid analytic spaces and it is a proper rigid analytic group such that the quotient map $T \rightarrow T / \Lambda$ is a homomorphism of rigid analytic groups (see pages 324-325 of [3]). Let $\operatorname{End}(T, \Gamma)$ denote the ring of endomorphisms of the algebraic group $T$ over $F$ such that $\phi(\Gamma) \subseteq \Gamma$. The operation of forming quotients induces an injective homomorphism

$$
h_{T, \Lambda}: \operatorname{End}(T, \Gamma) \longrightarrow \operatorname{End}(T / \Gamma)
$$

where the latter is the ring of rigid analytic endomorphisms of the rigid analytic group $T / \Lambda$.

THEOREM 3.2. The homomorphism $h_{T, A}$ is an isomorphism.

Proof. This is Satz 5 of [2] on page 33.

THEOREM 3.3. Let $F$ be a local field, let $T$ be a split torus over $F$ and let $\Lambda \subset T(F)$ be a discrete lattice. Then the quotient $T / \Lambda$ is isomorphic to the rigid analytic variety underlying an abelian variety over $F$ if and only if there is a homomorphism $\lambda$ from $\Lambda$ to the character group $\operatorname{Hom}\left(T, \mathrm{G}_{m}\right)$ of $T$ such that the bilinear map:

$$
(\alpha, \beta) \mapsto \lambda(\alpha)(\beta): \Lambda \times \Lambda \rightarrow F^{*}
$$

is symmetric and $\operatorname{ord}_{F}((\alpha, \alpha))>0$ whenever $1 \neq \alpha \in \Lambda$.

Proof. This is Theorem 5 of [3] on page 338.

Notation 3.4. For every field $F$ and for every commutative group scheme $B$ over $F$ let $\operatorname{End}(B)$ denote the ring of endomorphisms of $B$ as a group scheme over $F$. Moreover let $\operatorname{Aut}(B) \subseteq \operatorname{End}(B)$ denote the group of automorphisms of $B$ over $F$. Let $F$ be a local field, let $T$ be a split torus over 
$F$ and let $\Lambda \subset T(F)$ be a discrete lattice such that the quotient $T / \Lambda$ isomorphic to the rigid analytic variety underlying an abelian variety $B$ over $F$. By GAGA (see Theorem 2.8 of [7] on page 349) the ring of endomorphisms of $B$ as a group scheme over $F$ and the ring of rigid analytic endomorphisms of the rigid analytic group $T / \Lambda$ are the same. Let $g_{B}: \operatorname{End}(B) \rightarrow \operatorname{End}(C(T))$ denote the ring homomorphism which is the composition of

$$
h_{T, \Lambda}^{-1}: \operatorname{End}(B)=\operatorname{End}(T / \Lambda) \longrightarrow \operatorname{End}(T, \Lambda)
$$

and the forgetful map:

$$
\operatorname{End}(T, \Lambda) \longrightarrow \operatorname{End}(T)=\operatorname{End}(C(T)) .
$$

Finally let $f_{B}$ denote the Q-linear representation

$$
\left.\left(g_{B}\right)\right|_{\operatorname{Aut}(B)} \otimes \operatorname{id}_{Q}: \operatorname{Aut}(B) \rightarrow G L(C(T) \otimes Q) .
$$

LEMma 3.5. Let $F$ be a local field and let $\rho: G \rightarrow G L(V)$ be a representation of the finite group $G$ on a finite dimensional vector space $V$ over Q. Then there is a split torus $T$ over $F$ and a discrete lattice $\Lambda \subset T(F)$ such that the following holds:

(i) the quotient $T / \Lambda$ isomorphic to the rigid analytic variety underlying an abelian variety $B$ over $F$,

(ii) there is an injective homomorphism $\sigma: G \rightarrow \operatorname{Aut}(B)$,

(iii) the representation $\left(f_{B}\right) \circ \sigma$ is isomorphic to $\rho$.

Proof. Note that there is a $G$-submodule $\Gamma<V$ which is a finitely generated, free Z-module of rank $\operatorname{dim}(V)$ and spans $V$ as a vector space over Q. Let $T$ be a split torus over $F$ such that $C(T)$ is equal to $\Gamma$. Choose a uniformizer $\pi \in F^{*}$ and let $\Lambda<F^{*} \otimes C(T)$ be the abelian group generated by the set $\{\pi \otimes \gamma \mid \gamma \in C(T)\}$. Let $v: F^{*} \rightarrow Z$ be the valuation on $F$ normalised such that $v(\pi)=1$. Under the homomorphism $v \otimes \operatorname{id}_{C(T)}$ the group $\Lambda$ maps isomorphically onto its image which is $Z \otimes C(T)$. Therefore $\Lambda$ is a discrete lattice in $T(F)$.

Let $\langle\cdot, \cdot\rangle: V \times V \rightarrow \mathbb{Q}$ be a symmetric positive definite bilinear form. We may assume, by multiplying by a positive integer, if it is necessary, that $\langle\cdot, \cdot\rangle$ takes integer values on $C(T)=\Gamma$. Let $\theta: C(T) \rightarrow \operatorname{Hom}(C(T), Z$ ) be the homomorphism given by the rule $\theta(\alpha)(\beta)=\langle\beta, \alpha\rangle$ for every $\alpha, \beta \in C(T)$. Let $\lambda: \Lambda \rightarrow \operatorname{Hom}\left(T, \mathrm{G}_{m}\right)$ denote the composition:

$$
\Lambda \stackrel{v \otimes \operatorname{id}_{C(T)}}{\longrightarrow} Z \otimes C(T) \cong C(T) \stackrel{\theta}{\longrightarrow} \operatorname{Hom}\left(C(T), Z_{1}\right) \cong \operatorname{Hom}\left(T, \mathrm{G}_{m}\right) .
$$

The map $\lambda$ satisfies the conditions of Theorem 3.3, and hence the quotient 
$T / \Lambda$ is isomorphic to the rigid analytic variety underlying an abelian variety $B$ over $F$. Note that the natural action of $G$ on $F^{*} \otimes C(T)$ induced by the $G$-module structure on $C(T)=\Gamma$ leaves the subgroup $\Lambda$ invariant. Therefore the natural action of $G$ on $T$ descends down to the quotient $T / \Lambda$, that is, there is an injective homomorphism $\sigma: G \rightarrow \operatorname{Aut}(B)$ by GAGA. The objects $T, \Lambda, \sigma$ obviously satisfy condition (iii) above.

For every abelian variety $A$ defined over a field $F$ and for every prime number $l$ different from the characteristic of $F$ let $T_{l}(A)$ be the $l$-adic Tate module of $A$ and let $V_{l}(A)$ denote the Galois representation $T_{l}(A) \otimes_{Z_{l}} Q_{l}$ over $F$. For every group $G$, for every representation $\rho: G \rightarrow G L(V)$ on a vector space $V$ over $\mathrm{Q}$, and for every prime number $l$ let $\rho_{l}: G \rightarrow G L\left(V \otimes_{\mathrm{Q}} \mathrm{Q}_{l}\right)$ denote the $\mathrm{Q}_{l}$-linear extension of $\rho$.

Proposition 3.6. Let $F$ be a local field and let $\rho: \operatorname{Gal}(\bar{F} \mid F) \rightarrow G L(V)$ be a representation on a finite dimensional vector space $V$ over $\mathrm{Q}$ which is continuous with respect to the discrete topology on $G L(V)$. Then there is an abelian variety $A$ defined over $F$ such that for every prime number $l$ different from the characteristic of $F$ the $\mathrm{Gal}(\bar{F} \mid F)$-representation $V_{l}(A)$ has a quotient isomorphic to $\rho_{l}$.

Proof. Let $G$ be the image of $\operatorname{Gal}(\bar{F} \mid F)$ with respect to $\rho$ and by slight abuse of notation let $\rho$ denote the representation furnished by the inclusion $G \rightarrow G L(V)$. Let $T$ be a split torus over $F$ and let $\Lambda \subset T(F)$ be a discrete lattice which satisfies the properties of Lemma 3.5 with respect to the group $G$ and the representation $\rho$. As above let $B$ be an abelian variety over $K$ which is isomorphic to $T / \Lambda$ as a rigid analytic variety and let $\sigma: G \rightarrow \operatorname{Aut}(B)$ be a homomorphism which satisfies conditions (ii) and (iii) of Lemma 3.5.

Now let $l$ be a prime number different from the characteristic of $F$. The quotient map $i: T \rightarrow B$ induces an injective homomorphism $i_{l}: T_{l}(T) \rightarrow$ $T_{l}(B)$ where $T_{l}(T)$ is the Tate module of the torus $T$. The cokernel of $i_{l}$ is isomorphic to $C(T) \otimes_{\mathrm{z}} \mathrm{Z}_{l}$ equipped with the trivial Galois-action. Therefore there is a short exact sequence:

$$
0 \longrightarrow C(T) \otimes_{\mathrm{Z}} \mathrm{Q}_{l}(1) \longrightarrow V_{l}(B) \longrightarrow C(T) \otimes_{\mathrm{Z}} \mathrm{Q}_{l} \longrightarrow 0
$$

of $\operatorname{Gal}(\bar{F} \mid F)$-representations, where for every $Q_{l}[\operatorname{Gal}(\bar{F} \mid F)]$-module $W$ we let $W(1)$ denote the Tate twist of $W$, and we let $\operatorname{Gal}(\bar{F} \mid F)$ act on $C(T)$ trivially. The composition of $\rho: \operatorname{Gal}(\bar{F} \mid F) \rightarrow G$ and $\sigma$ furnishes a cohomology class in the pointed Galois cohomology set $H^{1}\left(F, \operatorname{Aut}\left(B_{\bar{F}}\right)\right)$. Let $A$ denote 
the twist of $B$ with respect to this class. Because the action of $G$ on $B$ lifts to $T$, its action on $V_{l}(B)$ respects the filtration of (3.6.1). Therefore there is a short exact sequence:

$$
0 \longrightarrow V \otimes_{\mathrm{Q}} \mathrm{Q}_{l}(1) \longrightarrow V_{l}(A) \longrightarrow V \otimes_{\mathrm{Q}} \mathrm{Q}_{l} \longrightarrow 0
$$

where $\operatorname{Gal}(\bar{F} \mid F)$ acts on $V$ via $\rho$. The claim is now clear.

For every variety $V$ defined over a field $F$ and for every extension $K$ of $F$ let $V_{K}$ denote the base change of $V$ to $\operatorname{Spec}(K)$. Recall that we say that an abelian variety $A$ defined over a local field $F$ has semi-stable reduction if the fibre over $\operatorname{Spec}\left(\boldsymbol{k}_{F}\right)$ of its Néron model over $\operatorname{Spec}\left(\mathcal{O}_{F}\right)$ is a semi-abelian variety. We will need the following fundamental result.

THEOREM 3.7. Let $F$ be a local field and let $A$ be an abelian variety defined over $F$. Let $l$ be a prime number different from the characteristic of $\boldsymbol{k}_{F}$. Then the following conditions are equivalent:

(i) the action of $I_{F}$ on $V_{l}(A)$ is unipotent,

(ii) the abelian variety $A$ has semi-stable reduction over $F$.

Proof. This follows from Proposition 3.5 of [4] on page 350 and Corollaire 3.8 of [4] on page 353 .

Proposition 3.8. There is a local field $F$ of characteristic zero with a perfect residue field and there is an abelian variety over $F$ which does not acquire semi-stable reduction over any solvable extension of $F$.

Proof. By Theorem 1.1 there is a local field $F$ of characteristic zero with a perfect residue field and there is a finite Galois extension $K \mid F$ such that there is no solvable extension $L \mid F$ such that the extension $K L \mid K$ is unramified, where $K L$ is the composite of $K$ and $L$. Choose a faithful finitedimensional Q-linear representation $\rho: \operatorname{Gal}(K \mid F) \rightarrow G L(V)$ and by slight abuse of notation let $\rho$ also denote the composition of the quotient map $\operatorname{Gal}(\bar{F} \mid F) \rightarrow \operatorname{Gal}(K \mid F)$ and $\rho$. Choose a prime number $l$ different from the characteristic of $\boldsymbol{k}_{F}$. By Proposition 3.6 there is an abelian variety $A$ over $F$ such that $V_{l}(A)$ has a quotient $W$ isomorphic to $\rho_{l}$ as a $\operatorname{Gal}(\bar{F} \mid F)$-representation.

We claim that $A$ does not acquire semi-stable reduction over any solvable extension of $F$. Assume the contrary and let $L \mid F$ a finite solvable Galois extension such that $A_{L}$ has semi-stable reduction. By Theorem 3.7 the action of $I_{L}$ on $V_{l}(A)$ is unipotent. Therefore the the action of $I_{L}$ on $W$ is 
unipotent, too. In particular the image of $I_{L}$ under $\rho_{l}$ is a pro- $l$ group, and hence the extension $\operatorname{Gal}(K L \mid L)$ is tamely ramified. Therefore there is a finite solvable Galois extension $L^{\prime} \mid L$ such that $\mathrm{Gal}\left(K L^{\prime} \mid L^{\prime}\right)$ is unramified by Lemma 4.6 of [10]. The extension $L^{\prime} \mid F$ is solvable as it is a tower of solvable extensions. This is a contradiction.

Proof of Corollary 1.2. By the proposition above there is a field $F$ of characteristic zero complete with respect to a discrete valuation whose residue field is perfect and there is an abelian variety $A$ over $F$ which does not acquire semi-stable reduction over any solvable extension of $F$. For any geometrically irreducible, smooth, projective curve $D$ we let $\operatorname{Jac}(D)$ denote the Jacobian of $D$. By Faltings's trick (see Theorem 10.1 of [8] on page 198) there is a smooth, projective, geometrically irreducible curve $C$ over $F$ such that there is a surjective homomorphism $\phi: \operatorname{Jac}(C) \rightarrow A$ of abelian varieties over $F$. We claim that $C$ does not acquire semi-stable reduction over any solvable extension of $F$. Assume the contrary and let $K \mid F$ a finite solvable Galois extension such that $C_{K}$ has semi-stable reduction. Then $\operatorname{Jac}(C)_{K}=\operatorname{Jac}\left(C_{K}\right)$ also has semi-stable reduction by Theorem 2.4 of [1] on page 89. Therefore the action of $I_{K}$ on $V_{l}\left(\mathrm{Jac}(C)_{K}\right)$ is unipotent by Theorem 3.7 where $l$ is a prime number different from the characteristic of $\boldsymbol{k}_{F}$. Because the homomorphism $\phi_{l}: V_{l}\left(\mathrm{Jac}(C)_{K}\right) \rightarrow V_{l}\left(A_{K}\right)$ induced by $\phi$ is surjective we get that the action of $I_{K}$ on $V_{l}\left(A_{K}\right)$ is unipotent, too. Therefore $A_{K}$ has semi-stable reduction by Theorem 3.7. This is a contradiction.

Acknowledgement. The author was partially supported by the EPSRC grant P19164.

\section{REFERENCES}

[1] P. Deligne - D. Mumford, The irreducibility of the space of curves of given genus, Publ. Math. Inst. Hautes Études Sci. 36 (1969), pp. 75-109.

[2] L. GERRITZEN, Über Endomorphismen nichtarchimedischer holomorpher Tori, Invent. Math. 11 (1970), pp. 27-36.

[3] L. GerRitzen, On non-archimedean representations of abelian varieties, Math. Ann. 196 (1972), pp. 323-346

[4] A. GrothendiecK - M. Raynaud, Modèles de Néron et monodromie, Groupes de monodromie en Géometrie Algebrique, I, II, Lecture Notes in Math. 288 (Springer, 1972), pp. 313-523.

[5] D. Harbater, Mock covers and Galois extensions, J. Algebra, 91 (1984), pp. 281-293. 
[6] Gy. Károlyi - A. PÁL, The cyclomatic number of connected graphs without solvable orbits, to appear J. Ramanujan Math. Soc. (2012).

[7] W. LÜtKeвонmert, Formal-algebraic and rigid-analytic geometry, Math. Ann. 286 (1990), pp. 341-371.

[8] J. Milne, Jacobian varieties, Arithmetic geometry (Storrs, Conn., 1984) (Springer, New York ,1986), pp. 167-212.

[9] A. PÁL, Solvable points on projective algebraic curves, Canad. J. Math. 56 (2004), pp. 612-637.

[10] A. PÁL, Solvable points on genus one curves over local fields, Journal of the Institute of Mathematics of Jussieu (2012).

Manoscritto pervenuto in redazione il 5 Marzo 2012. 